\title{
Advanced renal cell carcinoma associated with von Hippel-Lindau disease: A case report and review of the literature
}

\author{
LEI ZHANG ${ }^{1-3 *}$, BIN XU $^{1-3^{*}}$, YIDUO WANG ${ }^{1 *}$, CHUNHUI LIU ${ }^{2,3}, \mathrm{KAI} \mathrm{LU}^{1}$, YEQING HUANG $^{2,3}$, \\ NING LIU ${ }^{1}$, XIAOWEN ZHANG ${ }^{1-3}$, SHUQIU CHEN ${ }^{1}$ and MING CHEN ${ }^{1-3}$ \\ ${ }^{1}$ Department of Urology, Zhongda Hospital, ${ }^{2}$ Surgical Research Center, School of Medicine, \\ and ${ }^{3}$ Institute of Urology, Southeast University, Nanjing, Jiangsu 210009, P.R. China
}

Received July 31, 2014; Accepted April 13, 2015

DOI: $10.3892 / \mathrm{ol} .2015 .3279$

\begin{abstract}
The autosomal dominant hereditary disorder von Hippel-Lindau (VHL) disease is caused by a germline mutation in the VHL gene. The symptoms of VHL include hemangioblastoma of the central nervous system, retinal angiomas, visceral tumors and multiple visceral cysts. However, advanced renal cell carcinoma (RCC) occurs in few VHL patients at initial diagnosis; in addition, sporadic VHL disease with de novo germline mutation is rare. The current study reports the clinical case of a 33-year-old Asian male patient diagnosed with advanced bilateral multicentric renal carcinomas. The patient underwent radical nephrectomy with embolectomy of the right kidney for treatment of $\mathrm{T}_{3} \mathrm{~b}$-stage RCC and laparoscopic nephron-sparing surgery of the left kidney. Sunitinib was administered following histological diagnosis and during follow-up. Genetic analysis revealed a missense mutation, c.194C > G (p.Ser65Trp). In addition, genetic analysis of the patient's parents and brothers, who were unaffected, confirmed a diagnosis of de novo VHL disease. To the best of our knowledge, the present study reports the first known case of a sporadic de novo germline mutation of VHL at c.194C $>$ G. Current understanding of the molecular genetics and pathophysiology of VHL disease, as well as developments in surgical and target therapies for RCC have advanced in recent years; however, early detection through genetic screening and regular clinical surveillance of VHL disease patients and their families continues to be the primary basis for managing the disease.
\end{abstract}

Correspondence to: Dr Ming Chen, Department of Urology, Zhongda Hospital, Southeast University, 87 Dingjiaqiao Road, Nanjing, Jiangsu 210009, P.R. China

E-mail: mingchenseu@126.com

*Contributed equally

Key words: von Hippel-Lindau disease, sporadic, kidney cancer, genetic screening

\section{Introduction}

The autosomal dominant hereditary disorder von Hippel-Lindau (VHL) disease is caused by a germline mutation in the VHL gene. VHL is characterized by abundantly vascularized tumors in multiple organs; such VHL tumors may include hemangioblastoma of the retina and central nervous system (CNS), renal cell carcinoma (RCC), pancreatic tumors, pheochromocytoma, endolymphatic sac tumors, epididymal cystadenoma as well as cysts and cystadenoma in the kidney, pancreas, epididymis and broad ligament (1). The incidence of VHL disease has been estimated to be 1 in 36,000 live births in the Caucasian population, with gradually more apparent clinical manifestations occurring between $~ 18$ and 30 years of age (2). Clinical diagnostic criteria include a positive family history and at least one typical VHL tumor. For isolated cases, at least two typical manifestations, including one hemangioblastoma, are required for diagnosis (3). Cases of sporadic VHL disease are rare. Approximately $20 \%$ of VHL patients demonstrate negative family history, with disease resulting from a de novo mutation (4). The VHL gene has been mapped to chromosome 3 (3p25-26) (5). Identification of pathogenic germline VHL mutations has been reported to provide a reliable method for the confirmation of diagnosis (6). The current study reports a case of de novo VHL disease in a male patient with advanced bilateral multifocal RCC involvement. Written informed consent was obtained from the patient. The relevant literature is also reviewed.

\section{Case report}

History and examination. A 33-year-old man was admitted to Zhongda Hospital, Southeast University (Nanjing, China) with a two-month history of progressive right flank pain, accompanied by a one-month history of recurrent painless gross hematuria. The patient's previous medical history included urological surgery at the age of 23 years due to an epididymal cyst and an ophthalmologic surgery at 26 years of age for retinal detachment resulting from bilateral retinal angiomatosis, which was performed at Shuyang People's Hospital (Suqian, China). At the time of the present study, the 
patient had two children, a son and a daughter, who were in good health; his father, aged 65 years, and his mother, aged 62 years, were also in good health. The patient's two brothers, aged 40 and 37 years old, with one child each were all are in good health.

Contrast-enhanced computed tomography (CT) scanning and angiography were used to detect the presence of quasi-circular enhancing lesions in the middle of the left kidney and large irregular enhancing lesions in the right kidney, extending into the vena cava, that formed an embolus (Fig. 1A-C). In addition, multiple cysts in the pancreas and kidney were detected radiologically (Fig. 1A).

Surgery and post-surgical course. The patient underwent a radical nephrectomy with an embolectomy of the right kidney for treatment of $\mathrm{T}_{3} \mathrm{~b}$-stage RCC (Fig. 1D). After three months, he successfully underwent laparoscopic nephron-sparing surgery (NSS) of the left kidney (Fig. 1E). Histological analysis of the renal tissue revealed clear cell carcinomas (Fig. 1F). Sunitinib was administered (50 mg every day for four weeks) following histological diagnosis and during follow-up.

Genetic study. Peripheral blood samples from nine genetic relatives of the patient were subjected to VHL mutation analysis. Genomic DNA was isolated from peripheral blood leukocytes using the CW0541 Blood Gen Midi kit (CW Biotech Co., Ltd., Beijing, China). The entire coding sequence of the VHL gene, including exon/intron boundaries, was amplified by polymerase chain reaction (PCR) using primers (7) manufactured by Generay Biotech Co., Ltd. (Shanghai, China). The PCR products were directly sequenced by Nanjing Springen Biotech Co., Ltd. (Nanjing, China) using the Eppendorf 5331 MasterCycler Gradient Thermal Cycler (Eppendorf, Hamburg, Germany) under the following conditions: Denaturation at $95^{\circ} \mathrm{C}$ for $10 \mathrm{~min}$, followed by 35 cycles of $95^{\circ} \mathrm{C}$ for $30 \mathrm{sec}, 56^{\circ} \mathrm{C}$ for $30 \mathrm{sec}$ and $72^{\circ} \mathrm{C}$ for $30 \mathrm{sec}$, and a final extension step at $72^{\circ} \mathrm{C}$ for $10 \mathrm{~min}$. The mutations were confirmed by two-way DNA strand sequencing and manually compared. A germline mutation was detected in exon 1 of the VHL gene [c.194C>G (p.Ser65Trp)] in genomic DNA from the peripheral blood sample of the patient, while no mutation was detected in the peripheral blood of the patient's relatives (Fig. 2).

\section{Discussion}

In adults, RCC accounts for $\sim 3 \%$ of all cancers and $\sim 85 \%$ of all primary malignant kidney tumors (8). In addition, $\sim 5 \%$ of RCCs have a hereditary basis, of which VHL is the most common cause. Furthermore, $75 \%$ of RCCs have clear cell histology, which is hereditarily caused by VHL (9). RCC and cysts in VHL disease are characterized by multicentricity and bilateral location in $>75 \%$ of patients $(10,11)$. The mean age at presentation is 10-20 years earlier than the age reported for sporadic renal disease (12). Transition from a cyst to a solid lesion is rare; however, the lining epithelium may be dysplastic or may have characteristics of carcinoma-in-situ, resulting in RCC (11). The case reported in the current study presented with a typical triad of symptoms, including hematuria, flank pain and flank mass. However, cases with this 'triad syndrome' are more advanced and rare; RCC and cysts often remain asymp- tomatic for long intervals $(13,14)$. Thus, serial imaging of the kidneys for monitoring and early diagnosis has the potential to enhance overall patient outcome. The standard method for the detection of renal involvement in patients with VHL is contrast-enhanced abdominal CT imaging (15).

At present, no definitive guidelines are available with regard to the timing and surgical approach for these multicentric bilateral tumors. However, the primary aim of treatment should be cancer control, rather than cure, as well as the preservation of functional parenchyma in order to avoid the morbidity associated with renal or adrenal loss. A number of studies have reported that the majority of RCCs in VHL exhibit a low pathological grade (16), gradual growth (17) and do not metastasize at diameters of $<3 \mathrm{~cm}$ (18). Therefore, an individual renal lesion may be maintained under regular surveillance until it reaches $3 \mathrm{~cm}$ in diameter, at which point NSS is performed to balance the prevention of metastatic progression against the reduction of the number of interventions (19). However, studies have revealed that the majority of patients with VHL-associated RCC eventually develop local recurrence with the concomitant requirement for repeated renal surgery (16). Certain clinicians recommend open NSS as the standard of care for treating RCC in the setting of VHL disease due to the inability of laparoscopy to achieve reliable hypothermia, in addition to the prolonged ischemia and increased complication rates associated with this approach. However, based on our experiences, the large incision of the abdominal wall may be problematic, resulting in muscle injury, the development of hernias and cosmetic damage. Laparoscopic NSS, performed by experienced surgeons on selected patients, is an alternative to open surgery (20); the oncological outcome, based on available series with limited follow-up data, appears to be similar to the outcome achieved with open NSS (21). Furthermore, an increasing trend towards the use of percutaneous radiofrequency ablation or cryoablation for small RCCs has been observed as this method is less invasive compared with other therapies $(22,23)$. However, in rare cases with an advanced clinical stage of RCC in which the kidney cannot be preserved, such as that of the present study, or probable end-stage renal disease from the reiterated surgical treatments of localized tumors, total nephrectomy may be the only option due to a lack of alternative therapies. For patients who have undergone bilateral nephrectomy, renal replacement therapy may not be avoided $(24,25)$.

VHL disease occurs due to the inheritance of a mutated germline copy of the VHL gene from an affected parent. The wild type copy of the gene is then inactivated by a somatic event. However, $20 \%$ of patients with VHL develop the disease due to a de novo mutation. Inactivation of the wild type allele observed in tumors from VHL patients is consistent with the classic 'two hit' hypothesis of tumorigenesis $(26,27)$. Currently, $>400$ different mutations have been identified, including deletion, missense, nonsense, frameshift and insertion mutations (http://www.umd.be/VHL/W_VHL). In the present study, the germline mutation c.194C $>\mathrm{G}$ of the VHL gene was detected in the patient; it was predicted that this mutation may induce a serine-to-tryptophan substitution at amino acid position 65 (p.Ser65Trp) within the VHL protein (pVHL). Serine at amino acid position 65 is highly conserved among various species and a number of studies have demonstrated the 
A

B

C
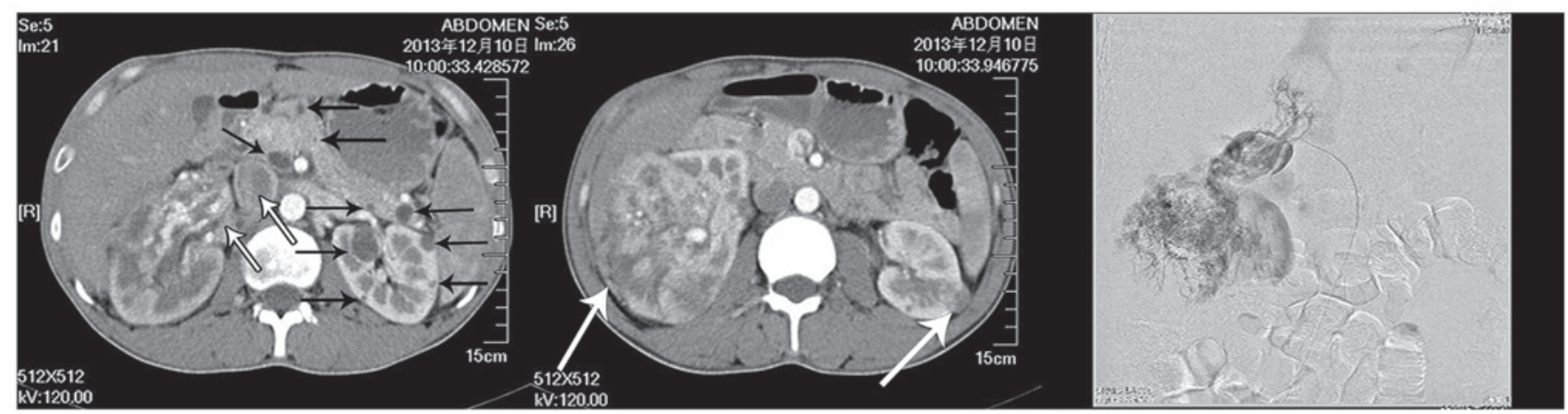

D

E

F
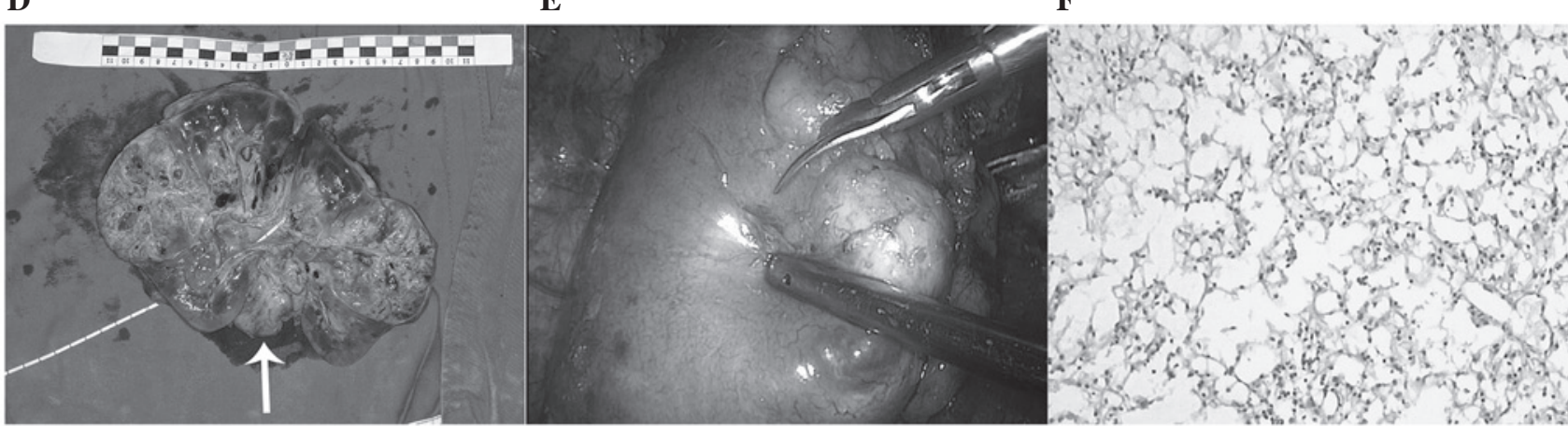

Figure 1. Clinical information collected from the patient. Axial computed tomography images showing: (A) Multiple cysts in the pancreas and kidney (black arrows) and embolus in the right kidney vein and vena cava (white arrows); and (B) quasi-circular enhancing lesions in the left kidney and huge irregular enhancing lesions in the right kidney (white arrows). (C) Angiography showing tortuosity of vessels in the right renal hilus and filling defect in vena cava. (D) Successful removal of the right renal tumor and embolus (white arrow). (E) Tumor and cysts in left kidney are revealed under laparoscopic vision. (F) Renal clear cell carcinoma was confirmed histologically (magnification, $\mathrm{x} 20$; hematoxylin and eosin stain).

\section{A}

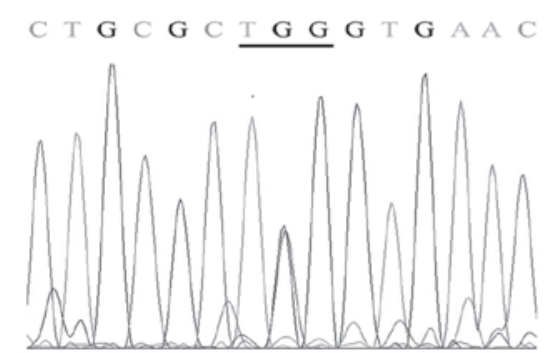

B

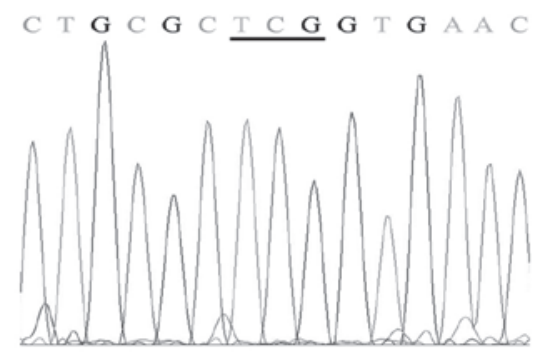

\section{I}

II

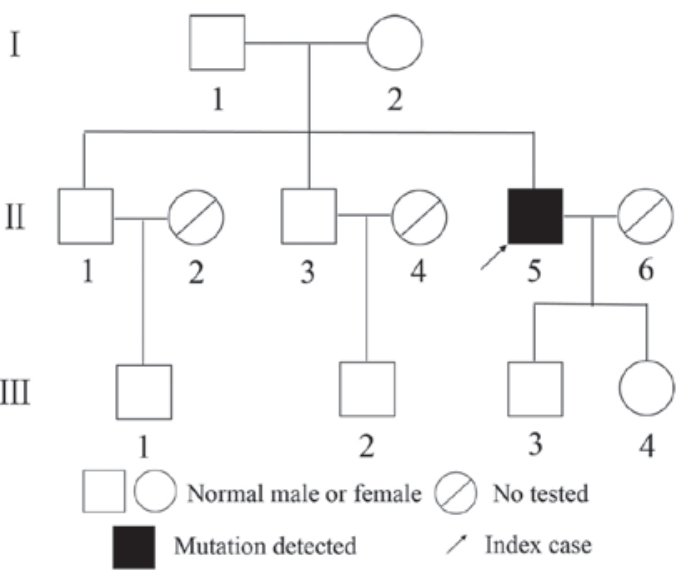

Figure 2. Resultant chromatogram profile following direct DNA sequencing of von Hippel-Lindau (VHL). (A) VHL c.194C $>$ G mutation was identified in peripheral blood sample of the patient and (B) normal VHL sequences were identified in peripheral blood samples from the patient's family members. (C) Pedigree displaying the sporadic development of VHL disease.

presence of mutations at this position (6,28-30). However, in previous cases involving this mutation, patients were reported to have a positive family history of VHL disease. In addition, no relatives of the patient in the current study were found to 
carry this mutation or had any clinical indications of VHL disease. Therefore, for the patient discussed in the present study, a de novo mutation may have occurred in a germ cell of either parent or at the embryo stage.

In conclusion, the current study presents a rare case of a de novo VHL patient with advanced RCC at first diagnosis; to the best of our knowledge, the patient discussed in the present study is the first known case of a sporadic de novo germline mutation of VHL at c.194C $>$ G. Current understanding of the molecular genetics and pathophysiology of VHL disease, as well as developments in surgical and target therapies for RCC have advanced in recent years; however, early detection through genetic screening and regular clinical surveillance of VHL disease patients and their families continues to be the predominant mode of disease management. The enhanced sensitivity of imaging techniques and advancements in therapeutic techniques, including radiofrequency ablation and NSS allow for the identification and management of tumors at earlier stages, thereby attenuating the morbidity and mortality rates associated with VHL disease.

\section{Acknowledgements}

The present study was supported by grants from the National Natural Science Foundation of China (nos. 81370849, 81300472, 81070592 and 81202034), the Natural Science Foundation of Jiangsu Province (nos. BL2013032 and BK2012336), the Natural Science Foundation of Nanjing City (no. 201201053), the Natural Science Foundation of Southeast University (no. 3290002402), the Science Foundation of Ministry of Education of China (no. 20120092120071) and the Fundamental Research Funds for the Central Universities and Graduate Innovative Foundation of Jiangsu Province (no. CXZZ13_0133).

\section{References}

1. Maher ER, Yates JR, Harries R, Benjamin C, Harris R, Moore AT and Ferguson-Smith MA: Clinical features and natural history of von Hippel-Lindau disease. Q J Med 77: 1151-1163, 1990.

2. Richard S, Graff J, Lindau J and Resche F: Von Hippel-Lindau disease. Lancet 363: 1231-1234, 2004.

3. Kaelin WG Jr: Molecular basis of the VHL hereditary cancer syndrome. Nat Rev Cancer 2: 673-682, 2002.

4. Neumann HP, Riegler P, Huber W, et al: The challenge of kidney lesions in von Hippel-Lindau disease. Contrib Nephrol 136: 193-207, 2001.

5. Latif F, Tory K, Gnarra J, et al: Identification of the von Hippel-Lindau disease tumor suppressor gene. Science 260: 1317-1320, 1993.

6. Stolle C, Glenn G, Zbar B, et al: Improved detection of germline mutations in the von Hippel-Lindau disease tumor suppressor gene. Hum Mutat 12: 417-423, 1998.

7. Chen J, Geng W, Zhao Y, et al: Clinical and mutation analysis of four Chinese families with von Hippel-Lindau disease. Clin Transl Oncol 15: 391-397, 2013.

8. Zhang L, Xu B, Chen S, et al: The complex roles of microRNAs in the metastasis of renal cell carcinoma. J Nanosci Nanotechnol 13 3195-3203, 2013.

9. Bradley S, Dumas N, Ludman M and Wood L: Hereditary renal cell carcinoma associated with von Hippel-Lindau disease: a description of a Nova Scotia cohort. Can Urol Assoc J 3: 32-36, 2009.
10. Malek RS, Omess PJ, Benson RC Jr and Zincke H: Renal cell carcinoma in von Hippel-Lindau syndrome. Am J Med 82: 236-238, 1987.

11. Choyke PL, Glenn GM, Walther MM, et al: The natural history of renal lesions in von Hippel-Lindau disease: A serial CT study in 28 patients. AJR Am J Roentgenol 159: 1229-1234, 1992.

12. Choyke PL, Glenn GM, Walther MM, Patronas NJ, Linehan WM and Zbar B: von Hippel-Lindau disease: Genetic, clinical and imaging features. Radiology 194: 629-642, 1995.

13. Schips L, Lipsky K, Zigeuner R, Salfellner M, Winkler S, Langner C, Rehak P, Pummer K and Hubmer G: Impact of tumor-associated symptoms on the prognosis of patients with renal cell carcinoma: A single-center experience of 683 patients. Urology 62: 1024-1028, 2003.

14. Ares Valdés Y: Correlation between symptoms and survival in patients with renal cell carcinoma. Arch Esp Urol 62: 201-206, 2009 (In Spanish)

15. Lonser RR, Glenn GM, Walther M, Chew EY, Libutti SK, Linehan WM and Oldfield EH: von Hippel-Lindau disease. Lancet 361: 2059-2067, 2003.

16. Steinbach F, Novick AC, Zincke H, et al: Treatment of renal cell carcinoma in von Hippel-Lindau disease: A multicenter study. J Urol 153: 1812-1816, 1995.

17. Neumann HP, Bender BU, Berger DP, et al: Prevalence, morphology and biology of renal cell carcinoma in von Hippel-Lindau disease compared to sporadic renal cell carcinoma. J Urol 160: 1248-1254, 1998.

18. Duffey BG, Choyke PL, Glenn G, Grubb RL, Venzon D, Linehan WM and Walther MM: The relationship between renal tumor size and metastases in patients with von Hippel-Lindau disease. J Urol 172: 63-65, 2004.

19. Herring JC, Enquist EG, Chernoff A, Linehan WM, Choyke PL and Walther MM: Parenchymal sparing surgery in patients with hereditary renal cell carcinoma: 10-year experience. J Urol 165: 777-781, 2001.

20. Gill IS, Kavoussi LR, Lane BR, et al: Comparison of 1,800 laparoscopic and open partial nephrectomies for single renal tumors. J Urol 178: 41-46, 2007.

21. Ljungberg B, Cowan NC, Hanbury DC, et al: EAU guidelines on renal cell carcinoma: The 2010 update. Eur Urol 58: 398-406, 2010.

22. Pavlovich CP, Walther M, Choyke PL, Pautler SE, Chang R, Linehan WM and Wood BJ: Percutaneous radio frequency ablation of small renal tumors: Initial results. J Urol 167: 10-15, 2002.

23. Gill IS, Remer EM, Hasan WA, et al: Renal cryoablation: Outcome at 3 years. J Urol 173: 1903-1907, 2005.

24. Goldfarb DA: Nephron-sparing surgery and renal transplantation in patients with renal cell carcinoma and von Hippel-Lindau. J Intern Med 243: 563-567, 1998.

25. Goldfarb DA, Neumann HP, Penn I and Novick AC: Results of renal transplantation in patients with renal cell carcinoma and von Hippel-Lindau disease. Transplantation 64: 1726-1729, 1997.

26. Knudson AG Jr: Genetics of human cancer. J Cell Physiol 129 (Suppl 4): 7-11, 1986.

27. Neumann HP and Zbar B: Renal cysts, renal cancer and von Hippel-Lindau disease. Kidney Int 51: 16-26, 1997.

28. Chen F, Kishida T, Yao M, et al: Germline mutations in the von Hippel-Lindau disease tumor suppressor gene: Correlations with phenotype. Hum Mutat 5: 66-75, 1995.

29. Crossey PA, Richards FM, Foster K, et al: Identification of intragenic mutations in the von Hippel-Lindau disease tumour suppressor gene and correlation with disease phenotype. Hum Mol Genet 3: 1303-1308, 1994.

30. Maher ER, Webster AR, Richards FM, Green JS, Crossey PA, Payne SJ and Moore AT: Phenotypic expression in von Hippel-Lindau disease: Correlations with germline VHL gene mutations. J Med Genet 33: 328-332, 1996. 